Ger J Exerc Sport Res 2017 · 47:301-304 DOI 10.1007/s12662-016-0433-0

Eingegangen: 21. April 2016

Angenommen: 19. Dezember 2016

Online publiziert: 13. Januar 2017

(c) Der/die Autor(en) 2017. Dieser Artikel ist eine Open-Access-Publikation.

CrossMark

\author{
Martin Schnitzer · Maximilian Bork · Theresa Eder · Nicole Zobl • \\ Philipp Schlemmer \\ Institut für Sportwissenschaft, Universität Innsbruck, Innsbruck, Österreich
}

Die Wirkungsforschung von Sportgroßveranstaltungen ist nach wie vor ein Kerngebiet der sportökonomischen Literatur. Es hat sich jedoch große Skepsis über die von Politiker/innen so häufig genannten positiven Effekte von Sportgroßveranstaltungen verbreitet (Kaiser 2016), wie aus den negativen Volksbefragungen zur Bewerbung von Olympischen Spielen (z. B. München 2022, Hamburg 2024) hervorgeht (Könecke et al. 2016). Der so oft verwendete Begriff "legacy“ (das Erbe einer Veranstaltung) wird missverständlich gebraucht (Preuss 2015), und die Messbarkeit von Wirkungen (z. B. Vermittlung von Werten, Aufbau eines Images, Völkerverständigung) ist methodisch nicht einfach. Da die Forschungsansätze im Bereich der nicht messbaren (intangiblen) Effekte dürftig sind, versucht dieser Beitrag am Beispiel der Youth Olympic Games (YOG) zu messen, welche Langzeitwirkungen diese Effekte aus Sicht von deutschen und österreichischen Athlet/innen aufweisen - und zwar 5 bzw. 3 Jahre nachdem sie an den Olympischen Jugendspielen 2010 und 2012 teilgenommen haben.

\section{Aktueller Stand der Literatur}

Die Durchführung einer Großveranstaltung kann viele Effekte mit sich bringen. Diese können positiver oder negativer Natur sein (Heinemann 1995) und beispielsweise über ökonomische Input-Output-Tabellen oder über Kosten-
Nutzen-Analysen gemessen werden (Parent und Smith-Swan 2013; Preuss 2004). Methodisch greifen diese Analysen jedoch häufig zu kurz, da intangible Effekte, die im Zuge von Veranstaltungen auftreten, keine oder zu wenig Berücksichtigung finden (Thöni 2014). Gerade bei Veranstaltungen wie den YOG spielen nicht quantifizierbare (intangible) Effekte eine besondere Rolle, zumal das Internationale Olympische Komitee (IOC) die Vermittlung von (olympischen) Werten in der Zielsetzung der YOG hervorhebt (IOC 2014). Zudem kann eine Gegenüberstellung von Kosten und Nutzen der YOG nicht über einen rein ökonomischen Ansatz gerechtfertigt werden, wie das Beispiel der YOG 2012 zeigt (Schnitzer et al. 2016).

Obwohl die YOG bereits wissenschaftlich analysiert wurden (Hanstad et al. 2014), ist der Analyse intangibler Effekte dieser Veranstaltung kaum Beachtung geschenkt worden. Zwar haben Schnitzer et al. (2014), Peters und Schnitzer (2015) sowie Kristiansen (2013) die Wirkungen der YOG auf die teilnehmenden Athlet/innen am Beispiel der YOG 2012 in Innsbruck untersucht; einer wissenschaftlichen Betrachtung nicht quantifizierbarer Wirkungen der YOG auf einzelne Stakeholder (mit mehreren Jahren Abstand zur Veranstaltung) ist bis dato aber keine Bedeutung zugemessen worden.

Eine der Herausforderungen im Allgemeinen und im Speziellen für diese
Arbeit stellt die Operationalisierung von intangiblen Effekten dar. Diese kann einerseits über die Vision und Zielsetzungen und andererseits über die Besonderheiten der YOG erfolgen. Die Vision der YOG strebt die Weitervermittlung des olympischen Gedankenguts (Streben nach Frieden und Humanität durch den Sport) über die Athlet/innen (in der Form von Botschafter/innen) an (Hofmann 2013). Mit Hilfe des „Culture and Education Programme" (CEP) sollen die YOG außerdem den Bildungsgedanken aufgreifen und den Austausch unter den jungen Athlet/innen fördern. Studien haben jedoch ergeben, dass dem Wettkampfgedanken auch bei den YOG eine höhere Wichtigkeit gegenüber dem CEP gegeben wird (Peters und Schnitzer 2015; Schnitzer et al. 2014; Kristiansen 2013; Krieger 2012; Wong 2012). Die Literatur zeigt somit in mehrfacher Hinsicht ein heterogenes Bild, was die Teilnahme am und die Eindrücke und Erfahrungen vom CEP aus Sicht der Athlet/innen anbelangt. Die YOG werden für die teilnehmenden Athlet/innen primär als Motivationsschub für die sportliche Karriere angesehen, dienen aber auch zum Aufbau von (persönlichen) Netzwerken (Peters und Schnitzer 2015; Schnitzer et al. 2014). Die Studien zeigen zudem Schwierigkeiten, wie die Abhängigkeit von individuellen Wettkampf- und Zeitplänen der teilnehmenden Sportler/ innen auf. 


\begin{tabular}{lllll}
\hline Tab. 1 & \multicolumn{2}{c}{ Stichprobe der Untersuchung } & & \\
\hline & Singapur 2010 & Innsbruck 2012 & Gesamt \\
\hline $\begin{array}{l}\text { Grundgesamtheit } \\
\text { (Athlet/innen DOSB/ÖOC) }\end{array}$ & $n=133$ & $n=85$ & $n=218$ \\
\hline $\begin{array}{l}\text { Stichprobe } \\
\text { (Athlet/innen DOSB/ÖOC) }\end{array}$ & $\begin{array}{l}n=38 \\
\text { W: } 22 ; m: 15\end{array}$ & $\begin{array}{l}n=52 \\
\text { MW: } 21,1 ; \text { SD: } 0,8\end{array}$ & MW: 18,$9 ;$ SD: 1 & $\begin{array}{l}n=90(43 \%) \\
\text { W: } 52 ; m: 38\end{array}$ \\
& MW: 20,$2 ;$ SD: 3,5
\end{tabular}

Abseits der Erkenntnisse der YOG 2012 (Peters und Schnitzer 2015; Schnitzer et al. 2014) ist die Bedeutung der Teilnahme an Großveranstaltungen im Jugendalter für den weiteren Karriereverlauf der Jungathlet/innen wenig erforscht. Schwerpunkte in der Forschung von Karriereverläufen jugendlicher Athlet/innen liegen nach wie vor in der Betrachtung der Rolle von schulischer/ universitärer Ausbildung und Spitzensport (Lally und Kerr 2013; Brown und Hartley 1998) oder der unterschiedlichen Wahrnehmung von Events auf Basis soziodemographischer Merkmale. So zeigen sich beispielsweise geschlechtsspezifische Unterschiede hinsichtlich Selbst- und Aufgabenorientierung sowie egoassoziierter und aufgabenbezogener Parameter (Murcia et al. 2008).

Auf Basis der Erkenntnisse von Befragungen der Athlet/innen, die im Rahmen vergangener YOG durchgeführt wurden, wird mit der vorliegenden Arbeit eine weitere Forschungslücke geschlossen, indem ehemalige Athlet/innen mehrere Jahre nach ihrer Teilnahme an den YOG über ihre „legacies“ Auskunft geben. Besonderes Augenmerk wird dabei auf die Rolle der YOG im Rahmen der sportlichen Karriere der Athlet/innen gelegt.

\section{Methode}

Die vorliegende Studie hat durch ihren Ex-post-Ansatz einen Pilotcharakter und aufgrund der schweren Erreichbarkeit der ehemaligen YOG-Teilnehmer/ innen wurde beschlossen, einen Online-Fragebogen zu entwickeln, der von dieser Zielgruppe 5 bzw. 3 Jahre nach Durchführung der YOG 2010 bzw. 2012 ausgefüllt werden sollte.

Die Erhebung wurde über die OnlineBefragungsplattform SoSci Survey im Zeitraum vom 16.11.2014 bis 31.01.2015 durchgeführt. Der Link zum Fragebogen wurde mit Hilfe des Deutschen Olympischen Sportbundes (DOSB) und des Österreichischen Olympischen Comités (ÖOC) an die jeweiligen Delegationen von Singapur und Innsbruck per E-Mail mit der Bitte um Teilnahme versandt. Darüber hinaus wurden die Athlet/ innen im Januar 2015 noch einmal per E-Mail an die Teilnahme an der Studie erinnert. Der Fragebogen (wird auf Anfrage von den Autoren bereitgestellt) war ausschließlich in deutscher Sprache verfügbar.

Der Fragebogen bestand insgesamt aus 23 Fragen, die das soziodemographische Profil der Athlet/innen, spezifische Aspekte zur Teilnahme an den Wettkämpfen, theoriebasierte Fragenblöcke, die sich auf die oben dargestellte Literatur und im Speziellen auf die von Schnitzer et al. (2014) durchgeführten Befragungen (während der YOG) stützten, den sportlichen Werdegang nach den YOG, Erinnerungen an die YOG und die Teilnahme an den CEP-Veranstaltungen mittels 5- bzw. 10-stufiger Likert-Skala abfragten. Die Stichprobe der Untersuchung wird in - Tab. 1 der Grundgesamtheit gegenübergestellt.

Für die statistische Analyse wurde das Statistikprogramm SPSS 18.0 herangezogen. Nach Prüfung der Normalverteilung (K-S-Test) und Prüfung auf Gleichheit der Fehlervarianzen (Levene-Test) wurden ungepaarte t-Tests zur Unterschiedsprüfung herangezogen. Alle Resultate wurden als absolute bzw. relative Häufigkeiten und Mittelwerte \pm Standardabweichung angegeben. Das Signifikanzniveau wurde mit $p \leq 0,05$ festgelegt, wobei Werte unter $p \leq 0,01$ als höchst signifikant angesehen wurden.

\section{Ergebnisse}

Die Frage nach den besten und schlechtesten Erinnerungen, die junge Athlet/ innen mit den YOG assoziieren, zeigt, dass die Eröffnungsfeier, der sportliche Erfolg, das Teamerlebnis und die Kulturerfahrung als beste Erinnerungen gesehen werden. Als schlechteste Erinnerungen werden am häufigsten die persönlichen Leistungen (Verletzungen bzw. Wettkampfleistung) sowie die Organisation vor Ort (Probleme mit Sicherheitsbestimmungen und Mängel in den Wettkampfstätten) genannt.

Der Großteil der Befragten (82\%) wertet die YOG als wichtigstes Event der Wettkampfsaison und $55 \%$ sehen die YOG sogar als die wichtigste Veranstaltung ihrer bisherigen sportlichen Karriere. $79 \%$ der Befragten geben an, die YOG-DNA (olympische Werte) in sich $\mathrm{zu}$ tragen und $57 \%$ behaupten, in den letzten 4 Wochen vor der Befragung über die YOG gesprochen $\mathrm{zu}$ haben. Außerdem fühlen sich $53 \%$ als Botschafter/in der YOG und geben den Olympischen Gedanken und die YOGWerte an andere weiter. $49 \%$ der Befragten haben die letzten YOG in Nanjing 2014 mitverfolgt, obwohl sie nicht daran teilgenommen haben. Hinsichtlich ihrer eigenen sportlichen Aktivität geben $58 \%$ an, noch immer Kadersportler/ innen zu sein. $27 \%$ der Befragten sind nicht mehr als Leistungssportler/innen aktiv, arbeiten aber immer noch im Sport. Insgesamt konnten sich $11 \%$ aller Befragten nach ihrer Teilnahme an den YOG auch für die Teilnahme an den Olympischen Spielen qualifizieren. Was die Teilnahme am CEP anbelangt, zeigt sich, dass die Teilnehmer/innen der YOG 2010 häufiger das CEP besucht haben als jene der YOG 2012.

Die Unterschiedsprüfungen ergeben, dass Athleten nach ihrer Teilnahme an den YOG signifikant häufiger eine Karriere im Profisport angetreten haben als Athletinnen (Athletinnen $=3,5 \pm 1,7$; Athleten $=4,5 \pm 1,0 ; p<0,01)$. Athletinnen, die sich als Profisportlerinnen sehen, trainieren signifikant mehr als Nichtprofisportlerinnen $(\mathrm{r}=0,7 ; p<0,01)$ und Kadersportlerinnen (Nichtkadersportlerinnen $=3,0 \pm 1,7 ;$ Kadersportlerinnen $=$ 
$4,5 \pm 1,1 ; p<0,01)$. Geschlechtsspezifische Unterschiede (Athleten $=4,7 \pm 0,6$; Athletinnen $=4,2 \pm 1,3 ; p<0,001)$ können auch hinsichtlich des Interesses für aktuelle Änderungen in der jeweiligen Sportart festgestellt werden. Dies gilt insbesondere für Kadersportler/innen (Nichtkadersportler/innen $=4,7 \pm 0,6$; Kadersportler/innen $=3,9 \pm 1,4 ; p<0,05$ ).

Der t-Test für unabhängige Stichproben ergibt, dass die Athleten ihre Trainingsorganisation nach den YOG signifikant häufiger änderten als die Athletinnen, während Letztere signifikant mehr Interesse an den YOG haben. Des Weiteren bestätigen sich die Professionalisierungstendenz bei den Kadersportlerinnen sowie ein höheres Interesse an den Olympischen Spielen bei den deutschen Athlet/innen. Österreichische Athlet/innen haben sich jedoch signifikant häufiger für die Olympischen Spiele qualifiziert als ihre deutschen Kolleg/innen.

Abschließend ist festzuhalten, dass die Befragten die YOG betreffend der Kategorien Erinnerung (z. B. Orte, Ereignisse, Begegnungen), Erfahrungen (z. B. internationales Teilnehmerfeld, sportlicher Erfolg, Training) sowie Wirkungen (sportliche, soziale und finanzielle Entwicklung) auf einer Likert-Skala von 1 (negativ) bis 10 (positiv) durchaus positiv bewerten (Erinnerungen: 9,58 - SD 0,79; Erfahrungen: 9,12 - SD 1,33; Wirkungen: 8,01 - SD 2,02).

\section{Diskussion und Schlussfolgerung}

Ziel vorliegender Studie ist es, die intangiblen Wirkungen der YOG aus Sicht von deutschen und österreichischen Athlet/ innen im Kontext des bisherigen Karriereverlaufs bzw. im geschlechtsspezifischen Kontext 5 bzw. 3 Jahre nach ihrer Teilnahme an den YOG 2010 und 2012 zu messen. Die Ergebnisse können wie folgt zusammengefasst und interpretiert werden:

\section{- YOG als außergewöhnliche Erfahrung:}

Die YOG waren für die teilneh-

menden Sportler/innen ein (wenn nicht das) Highlight ihrer sportlichen Karriere. Die Ergebnisse zeigen, dass Athleten/innen 5 bzw. 3 Jahre nach der Veranstaltung noch sehr positive
Erinnerungen an die YOG (2010 bzw. 2012) haben. Diese Ergebnisse decken sich mit den Befragungen während der YOG 2012 in Innsbruck (Schnitzer et al. 2014; Peters und Schnitzer 2015).

- YOG als Meilenstein im Leben der Sportler/innen: Die Mehrheit der Athlet/innen gibt an, dass die YOG die wichtigste Veranstaltung in ihrem Wettkampfkalender war. Dies bestätigt die Ergebnisse von Schnitzer et al. (2014), steht jedoch im Widerspruch zu den Ergebnissen von Kristiansen (2013) bzw. Krieger (2012). Hier liegt die Vermutung nahe, dass die Wichtigkeit eines Wettkampfs in der Karriere eines Sportlers/einer Sportlerin mit etwas zeitlichem Abstand besser eingeschätzt werden kann. Außerdem haben die YOG 77 \% der Befragten dazu animiert, mit dem Leistungssport weiterzumachen, d. h. solche Sportgroßevents können für die Athlet/innen durchaus als Motivationsspritze gesehen werden.

- Zeremonien der YOG als lebenslange Erinnerung: Der zeremonielle Charakter der YOG mit Eröffnungs-, Schluss- und Medaillenfeier scheint sich als Erinnerung stark in den Köpfen der Athlet/innen einzuprägen. Dies konnten auch Schnitzer et al. (2014) bzw. Peters und Schnitzer (2015) in den Befragungen während der YOG feststellen. Hier scheint sich ein großer Unterschied zwischen einer Multisportveranstaltung, wie es die YOG sind, und typischen Jugendweltmeisterschaften der Fachverbände aufzutun. Die Befragten erwähnen, dass die Kommunikation zwischen den Nationen, auch außerhalb der eigenen Sportart, eine besondere Erfahrung darstellt.

- Das CEP als Experimentierfeld: Das CEP (seit 2016 Learn and Share) war gerade in den ersten beiden Ausgaben der YOG $(2010,2012)$ unbekannt und viele Athlet/innen konnten aufgrund organisatorischer Schwierigkeiten (Logistik, Termindruck) nicht daran teilnehmen (Schnitzer et al. 2014; Kristiansen 2013). Das CEP konnte aber bestimmte Themenbereiche
Ger J Exerc Sport Res 2017 • 47:301-304

DOI 10.1007/s12662-016-0433-0

(c) Der/die Autor(en) 2017. Dieser Artikel ist eine Open-Access-Publikation.

\section{Schnitzer · M. Bork · T. Eder · N. Zobl . P. Schlemmer}

Intangible Effekte von Sportgroßveranstaltungen aus Sicht von Athlet/innen am Beispiel der Olympischen Jugendspiele 2010 und 2012

Zusammenfassung

Nachdem die Forschungsansätze im Bereich der nicht messbaren (intangiblen)

Effekte von Sportgroßveranstaltungen dürftig sind, versucht dieser Beitrag am Beispiel der Olympischen Jugendspiele (YOG) die Langzeitwirkungen der YOG aus Sicht von deutschen und österreichischen Athlet/innen, die an den YOG 2010 und 2012 teilgenommen haben, 5 bzw. 3 Jahre nach den YOG mittels einer Online-Befragung zu messen.

Schlüsselwörter

Effekt · Athlet/innen · Olympische Jugendspiele

Intangible "legacies" of majors sports events as perceived by athletes using the example of the 2010 and 2012 Youth Olympic Games

\section{Abstract}

Research on the intangible effects of major sports events is still poor. Therefore this article aims to analyze these effects, based on the Youth Olympic Games (YOG), as perceived by German and Austrian athletes having participated in the 2010 and 2012 YOG. An online survey was conducted 5 and 3 years respectively after the YOG took place.

Keywords

Impact · Athlete · Youth Olympic Games

wie den Kontakt mit sportlichen Vorbildern besonders fördern.

- Geschlechtsspezifische Unterschiede:

Die Unterschiedsanalysen zeigen geschlechtsspezifische Unterschiede hinsichtlich der Leistungsorientierung. Interessanterweise scheint jedoch das anhaltende Interesse an den YOG bei Athletinnen stärker ausgeprägt zu sein. Es ist auch festzuhalten, 
dass sich die höhere Leistungsorientierung der Athleten im Vergleich zu den Athletinnen in ihrem höheren Interesse für die Sportart, mögliche Regeländerungen oder technische Neuerungen niederschlägt. Diese Unterschiede konnten in anderen YOG-Studien nicht bestätigt werden, aber Murcia et al. (2008) zeigen diese Unterschiede hinsichtlich Selbst- und Aufgabenorientierung sowie egoassoziierter und aufgabenbezogener Parameter.

Aufgrund des Pilotcharakters der Studie wurden nur 2 Teilnehmerländer befragt. Dies führte zu einer kleinen Stichprobe und ist auch aufgrund der Eingrenzung der Befragten als Limitation anzusehen. Ebenso sind die Ergebnisse unter dem Vorbehalt zu betrachten, dass keine Differenzierung nach Sportarten vorgenommen wurde. Auf Basis der erlangten Ergebnisse konnte gezeigt werden, dass sowohl Erinnerungen als auch Wirkungen der YOG mehrere Jahre nach der Austragung immer noch sehr positiv bei den Athlet/innen verankert sind und als Meilenstein der sportlichen Karriere angesehen werden. Diese Erkenntnisse sollten in der Planung der Veranstaltung aus Sicht des Internationalen Olympischen Komitees, der lokalen Veranstalterkomitees, aber auch aus Sicht der Nationalen Olympischen Komitees (in der Zusammenstellung ihrer Mannschaften für die jeweiligen YOG) berücksichtigt werden. Wesentlich scheint auch, dass junge Athlet/ innen im Zuge ihrer Karriere auf Sportgroßereignisse entsprechend vorbereitet und anschließend nachbetreut werden (z. B. Umgang mit Erfolg/Misserfolg, Einordnung der Leistungen). Diese Studie kann als wichtiger Test für weitere Studien herangezogen werden, um die Wirkungen von Sportgroßveranstaltungen auf die jugendlichen Sportler/innen besser beleuchten zu können.

\section{Korrespondenzadresse}

\section{Schnitzer}

Institut für Sportwissenschaft, Universität Innsbruck

Fürstenweg 185, 6020 Innsbruck, Österreich Martin.Schnitzer@uibk.ac.at

Open access funding provided by University of Innsbruck and Medical University of Innsbruck.

Interessenkonflikt. M. Schnitzer, M. Bork, T. Eder, N. Zobl und P. Schlemmer geben an, dass kein Interessenkonflikt besteht.

Open Access Dieser Artikel wird unter der Creative Commons Namensnennung 4.0 International Lizenz (http://creativecommons.org/licenses/by/4.0/deed. de) veröffentlicht, welche die Nutzung, Vervielfältigung, Bearbeitung, Verbreitung und Wiedergabe in jeglichem Medium und Format erlaubt, sofern Sie den/die ursprünglichen Autor(en) und die Quelle ordnungsgemäßnennen, einen Linkzur Creative Commons Lizenz beifügen und angeben, ob Änderungen vorgenommen wurden.

\section{Literatur}

Brown, C., \& Hartley, D. L. (1998). Athletic identity and career maturity of male college student athletes. International Journal of Sport Psychology, 29, 17-26.

Hanstad, D. V., Parent, M. M., \& Houlihan, B. (2014). The youth olympic games. New York: Routledge.

Heinemann, K. (1995). Einführung in die Ökonomie des Sports: Ein Handbuch.Schorndorf:KarlHofmann.

Hofmann, A. R. (2013). Olympia als Bildungsidee: Beiträge zur olympischen Geschichte und Pädagogik. Wiesbaden:Springer.

IOC International Olympic Committee (2014). Factsheet 1 of 4: The YOG - vision and principles. http://www.olympic.org/documents/ reference_documents_factsheets/the_youth olympic_games.pdf.Zugegriffen:16.Okt.2014.

Kaiser, S. (2016). Sozioökonomie Olympischer Spiele. Sportwissenschaft, 46(14), 14.

Könecke, T., Schubert, M., \& Preuss, H. (2016). (N)Olympia in Germany? An analysis of the referendum against Munich 2022. Sportwissenschaft, 46, 15-24.

Krieger, J. (2012). Fastest, highest, youngest? Analysing the athlete's experience of the Singapore Youth Olympic Games. International Review for the Sociology of Sport, 48(6), 706-719.

Kristiansen, E. (2013). Competing for culture: Young Olympians' narratives from the first winter Youth Olympic Games. International Journal of Sport and Exercise Psychology doi:10.1080/1612197X. 2012.756259.

Lally, P.S., \& Gretchen, A. K. (2005). The career planning, athletic identity, and student role identity of intercollegiate student athletes. Research Quarterly for Exercies and Sport, 76(3), 275-285.

Murcia, J. A. M., Gimeno, E.C., \& Coll, D. G.-C. (2008) Relationships among goal orientations, motivational climate and flow in adolescent athletes: differences by gender. The Spanish Journal of Psychology, 11(1), 181-191.
Parent, M.M., \& Smith-Swan, S. (2013). Managing major sports events: theory and practice. New York: Routledge.

Peters, M., \& Schnitzer, M. (2015). Athletes' expectations, experiences and legacies of the Winter Youth Olympic Games Innsbruck 2012. Journal of Convention \& Event Tourism, 16(2), 116-144.

Preuss, H. (2004). Kosten-Nutzen-Analysen sportlicher Großveranstaltungen. In A. Krüger \& A. Dreyer (Hrsg.), Sportmanagement - Eine Themenbezogene Einführung (S. 169-192). München: Oldenbourg Wissenschaftsverlag.

Preuss, H. (2015). A framework for identifying the legacies of a mega sport event. Leisure Studies, 34(1), 643-664. doi:10.1080/02614367.2014. 994552.

Schnitzer, M., Peters, M., Scheiber, S., \& Pocecco, E. (2014). Perception of the culture and education programme of the Youth Olympic Games by the participating athletes: A case study for Innsbruck 2012. The International Journal of the History of Sport, 31(9), 1178-1193.

Schnitzer, M., Bodner, B., Scheiber, S., Thöni, E., \& Kopp, M. (2016). Konsumverhalten von Eventbesuchern der Olympischen JugendWinterspiele am Beispiel Innsbruck 2012. Sportwissenschaft, 46, 26-33.

Thöni, E. (2014). The legacy of the Innsbruck Winter Youth Olympic Games 2012. In D. V. Hanstad, M. Parent \& B. Houlihan (Hrsg.), The Youth Olympic Games. New York: Routledge.

Wong, D. (2012). Expect the unexpected? Anevaluation of the Singapore 2010 Youth Olympic Games. Journal of Policy Research in Tourism, Leisure and Events, 4(2), 138-154. 